\title{
ANOTHER BIRTHDAY ATTACK
}

Don Coppersmith

IBM Research

Yorktown Heights, NY 10598

Abstract: We show that a neet-in-the-middle attack can successfully defraud the Davies-Price message authentication scheme. Their scheme used message blocks in an iterated encipherment of an initial block, and it went through the message blocks twice, in order to prevent just such a "birthday" attack.

\section{Background}

This note concerns methods for attaching a digital signature to a long message. There are several proposals for hashing the long message into a shorter bashed value, which can then be digitally signed by a more expensive technique, for example RSA. [RSA] This allows the signature to be publicized without revealing the content of the message; it allows a shorter signature; and it decreases the computation time necessary for computing or checking signatures. [Den]

Rabin [Rab] introduced a scheme, based on a general block cipber. It can be described in terms of DES, although Rabin's proposal did not use DES. In this scheme, the message $M$ would be broken into 56-bit blocks $M_{j}$, and these message blocks would be used as keys for the iterated encipherment of some initial value $H_{0}$. The final encipherment, along with the initial value, would form the hash value. Thus

$$
\begin{aligned}
& H_{0}=\text { random } \\
& H_{i}=E_{M_{i}}\left(H_{i-1}\right), \quad 1 \leq i \leq n \\
& \text { RSA-Sign }\left(H_{0}, H_{i 2}\right) .
\end{aligned}
$$

(Notation: here and throughout, $E_{K}(X)$ is the DES encipberment of the cleartext $X$ under the key $K ; D_{K}(Y)$ is the DES decipherment of the ciphertext $Y$ under the key $K$.)

The problent with this scheme in conjunction with DES, is a "meet-in-the-middle" or "birthday" attack. The opponent, knowing the RSA-signature of the pair $\left(H_{0}, H_{n}\right)$ arising from sume legitimate message $M^{\prime}$, can devise a message $M$ whose content is largely selected by the opponent, but whose hash value is also $\left(H_{0}, H_{n}\right)$. Thus the RSA-signature of $\left(H_{0}, H_{n}\right)$ can be reused to sign this bogus message.

To accomplish this, the opponent need only evaluate $2^{33}$ enciphements, instead of the $2^{64}$ required by the naive trial-and-error approach. (He also uses $2^{32}-4 \times 10^{9}$ storage) Namely, the opponent specifies values of $M_{1}, M_{2}, \ldots, M_{n-2}$. Using the given value of $H_{0}$, he computes successively $H_{1}, H_{2}, \ldots, H_{n-2}$. Then for each of $2^{32}$ trial values $X$ for the message block $M_{n-1}$, he computes that value $H_{n-1}[X]=E_{X}\left(H_{n-2}\right)$ which $H_{n-1}$ would have if $X$ were chosen for $M_{n-1}$. These $2^{32}$ values are sorted and stored. Now for each of $2^{32}$ trial values $Y$ for the message block $M_{n}$, he computes that value $H_{n-1}^{\prime}[Y]=D_{Y}\left(H_{n}\right)$ which $H_{n-1}$ would need to have in order for $H_{n}$ to bave its correst value, under the assumption that $Y$ were chosen for $M_{n}$. Each of these values is compared against the sorted 
values for $H_{n-1}[X]$. If a match is found $\left(H_{n-1}[X]-H_{n-1}^{\prime}[Y]\right)$ then the assignments $M_{n-1}=X, M_{n}=Y$ complete the message $M$ to one satisfying our requirements. Finally, the expected number of "successful" pairs $(X, Y)$ is 1 , so that we will find one with reasonable probability; this probability can be increased by a modest increase in the work factor.

\section{The Davies-Price Scheme}

Davies and Price [DP] introduced another DES-based message authentication scheme, by which they hoped to avoid this attack. Their scheme differs from Rabin's in that they cycle through the message blocks twice. Thus,

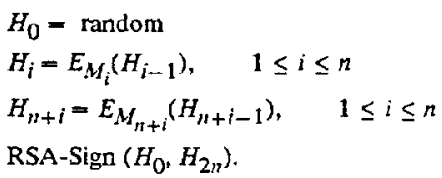

In the present note, we mount an attack on this scheme, similar to the meet-in-the-middle attack described above, with not much larger computational requirements.

\section{The Attack}

Our attack has two phases: a precomputation phase, which can be done once and used against all messages; and a stage tailored to the individual message. The requirements: for the precomputation stage, $2^{37}$ encipherments and $2^{36}$ storage; for the individual message, $2^{35}$ encipherments and $2^{32}$ storage. There are modest trade-offs available.

The message format is as follows. We select most of the message (say blocks $M_{19}$ through $M_{n}$ ) to be the text of that bogus message which we are trying to authenticate. Blocks $M_{1}$ and $M_{2}$ are chosen (by a meet-in-the-middle step) to put ourselves into a standardized position. Finally, blocks $M_{3}, M_{4}, \ldots, M_{18}$ are chosen, from among possibilities eaumerated during the precomputation, to "meet in the middle" one last time.

During the precomputation, we select an arbitrary 64-bit quantity $Z$, which is going be the value of $H_{2}, H_{4}, H_{6}, \ldots$, and $H_{18}$. We select $2^{36}$ trial values $X_{1}$ compute the values $E_{X}(Z)$, and sort and store these values. Now select $2^{36}$ trial values $Y$, compute the values $D_{Y}(Z)$, and compare each against the values $E_{X}(Z)$. Record each match: $E_{X}(Z)=D_{Y}(Z)$. We expect to find about 256 such pairs $\left\{\left(X_{i}, Y_{i}\right), 1 \leq i \leq 256\right\}$; if not, examine a few more values of $Y$. Each such pair $\left(X_{i}, Y_{i}\right)$ can be used as a message pair $\left(M_{3}, M_{4}\right),\left(M_{5}, M_{6}\right), \ldots$, or $\left(M_{17}, M_{18}\right)$, in the sense that if we have $H_{2}=Z, M_{3}=X_{i}, M_{4}=Y_{i}$, then we get $H_{4}=Z$.

Given a message $M^{\prime}=\left(M_{19}, M_{20}, \ldots, M_{n}\right)$, an RSA-signature of some pair $\left(H_{0}, H_{2 n}\right)$, the chosen value of $Z$ and the 256 pairs $\left(X_{i}, Y_{j}\right)$ gotten during precomputation, our task is to select values of $M_{1}, M_{2}, \ldots, M_{18}$ which will make $\left(H_{0}, H_{2 n}\right)$ a valid hash of $M=\left(M_{1}, M_{2}, \ldots, M_{n}\right)$.

First we find values of $M_{1}$ and $M_{2}$ such that $E_{M_{1}}\left(H_{0}\right)=D_{M_{2}}(Z)$; this takes $2^{33}$ encipherments and $2^{32}$ storage. We know that $H_{2}=Z$, so as long as the pairs $\left(M_{3}, M_{4}\right), \ldots,\left(M_{17}, M_{18}\right)$ are chosen from our list $\left(X_{i}, Y_{j}\right)$, we will have $H_{4}=H_{6}=\cdots=H_{18}=Z$. Assuming $H_{18}=Z$, use the values $M_{19}$ through $H_{n}$ to compute the value $H_{n} ;$ with the 
values $M_{1}$ and $M_{2}$ we can then get the value of $H_{n+2}$. Working backwards from $H_{2 m}$ using the values $M_{n}, M_{n-1}, \ldots, M_{19}$, we find the value of $H_{n+18}$

Now we use the precomputed pairs $\left(X_{j}, Y_{i}\right)$. For each of $256^{4}=2^{32}$ choices of four pairs $\left(X_{i}, Y_{i}\right)$ to be the values of $\left(M_{3}, M_{4}\right),\left(M_{5}, M_{6}\right),\left(M_{7}, M_{8}\right),\left(M_{9}, M_{10}\right)$, compute the value of $H_{n+10}$ that would result. (The efficient way to do this is to nun through the pairs lexicographically, so as not to recompute $E_{X_{i}}\left(H_{n+2}\right)$ for each of $2^{24}$ occurrences.) Sort and store these trial values of $H_{n+10}$. Similarly, select pairs to be the values of $\left(M_{11}, M_{12}\right),\left(M_{13}, M_{14}\right)$, $\left(M_{15}, M_{16}\right),\left(M_{17}, M_{18}\right)$, and compute backwards from $H_{n+18}$ to get trial values of $H_{n+10}$. Compare against the stored trial values. We expect one match, and the corresponding values of $M_{3}$ through $M_{13}$ finish our task.

\section{Extensions}

The Davies-Price scheme could be altered by running through the message three times instead of twice. This attack will still work, at the expense of a large increase in the number of "constrained" message blocks (the message blocks chosen by the algorithm, rather than selected by the user).

Another possible scheme would be to set up two initializing vectors.

$$
\begin{aligned}
& H_{0}, \quad H_{0}^{\prime}=\text { random } \\
& H_{i}=E_{M_{i}}\left(H_{i-1}\right), \quad 1 \leq i \leq n \\
& H_{i}^{\prime}-E_{M_{i}}\left(H_{i-1}^{\prime}\right), \quad 1 \leq i \leq n \\
& \text { RSA-Sign }\left(H_{0}, H_{n},{H^{\prime}}_{0}, H_{n}^{\prime}\right)
\end{aligned}
$$

Minor modifications to the present attack allow this scheme to be broken as well. Namely, do the same precomputation as before, and compute $M_{1}, M_{2}$ as before. Work forwards to find $H_{n-2}$, then use a meet-in-the-middle step to discover values $M_{n-1}, M_{n}$ which satisfy the requirement on $H_{n}$. Then the values $M_{3}$ through $M_{18}$ can be selected as before (from the pairs $\left(X_{i}, Y_{i}\right)$ ) to satisfy the requirements on $H^{\prime}{ }_{n}$

A word about "constrained" message blocks: since we only need to examine $2^{36}<23^{8}$ values $X$ in the preconputation, we can select them to be EBCDIC representations of alphanumeric characters, so that even the "constrained" message blocks needn't look like total nonsense. In fact, at the risk of increasing the number of such blocks, we can increase their plausibility, to the point of having a set English text with the freedom of choice made by substitution of synonyms. [DP]

\section{Trade-offs}

The presentation here tried to minimize computation time. There are two trade-offs available, which increase the computation time but decrease (1) storage and (2) length of constrained message, respectively.

When running a meet-in-the-middle attack, we work forward with $J$ values, sort and store the outcomes; work backwards with $K$ values, and compare against the $J$ values stored. We are likely to succeed if $J K \geq N$, where $N$ is the size of the space (in our case $2^{64}$ ). Thus we trade off storage of $J$ against computation time of $K$, subject to $K \geq J . J K \geq N$. 
In the present attack, we had 18 blocks of constrained message. This can be decreased if we are willing to spend more time in precomputation. A precomputation of $2^{41}$ encipherments and $2^{40}$ temporary storage would allow us to recover $2^{16}=65536$ pairs $\left(X_{i}, Y_{i}\right)$, and with that larger selection we would need to add only ten constrained message blocks: two at the beginning as before, and four pairs $\left(M_{3}, M_{4}\right), \ldots,\left(M_{9}, M_{10}\right)$ to allow the last meet-in-the-middle step to go through $\left(65536^{4}=2^{64}=N\right.$.)

\section{References}

[Den] D.E. Denning, Protecting public keys and signature keys, IEEE Computer, 1983, 16(2):27.

[DP] D.W. Davies and W.L. Price, "The Application of Digital Signatures based on Public Key Cryptosystems," NPL Report DNACS 39/80, National Physical.Laboratory, Teddington, Middlesex, England, Dec. 1980.

[Rab] M. Rabin, Digital Signatures, in "Foundations of Secure Computation," Academic Press, New York, 1978.

[RSA] R.L. Rivest, A. Shamir, and L. Adleman, "A Method for Obtaining Digital Signatures and Public-Key Cryptosystems," Conm. ACM, vol. 21, no. 2, Feb. 1978, pp. 120-126. 